\title{
Wigner Functions and Quark Orbital Angular Momentum
}

\author{
Asmita Mukherjee ${ }^{1, a}$, Sreeraj Nair ${ }^{1, b}$, and Vikash Kumar Ojha ${ }^{1, c}$ \\ ${ }^{1}$ Department of Physics, Indian Institute of Technology Bombay, Powai, Mumbai 400076, India
}

\begin{abstract}
Wigner distributions contain combined position and momentum space information of the quark distributions and are related to both generalized parton distributions (GPDs) and transverse momentum dependent parton distributions (TMDs). We report on a recent model calculation of the Wigner distributions for the quark and their relation to the orbital angular momentum.
\end{abstract}

\section{Introduction}

A fundamental question in hadronic physics is to understand how the spin of the nucleon is divided among the quarks and gluons that form the nucleon. In the EMC experiment, it was found that only a small fraction of the nucleon spin is carried by the quarks and antiquarks. Recent experiments suggest that the intrinsic spin carried by the gluons is also small. Thus a substantial part of the spin comes from quark and gluon orbital angular momentum (OAM). There are issues related to the gauge invariance and experimental measurability that complicates the understanding of the OAM. However, recently it was shown that the quantum mechanical Wigner distributions of quarks inside the nucleon can give information on the OAM carried by the quarks. Wigner distributions can be thought of as so-called quantum mechanical phase space distributions which give a joint position and momentum space information about the quarks in the nucleon. As position and momentum operators do not commute in quantum mechanics, they cannot be simultaneously determined. As a result, Wigner distributions are not positive definite. However, a reduced Wigner distribution can be defined after integrating over several variables, and these are positive definite. The

\footnotetext{
ae-mail: asmita@phy.iitb.ac.in

be-mail: sreeraj.phy@gmail.com

ce-mail: ohjavikash@gmail.com
}

Wigner distributions are related to the generalized parton correlation functions (GPCFs) or generalized transverse momentum dependent parton distributions (GTMDs). These are the mother distributions of the GPDs and TMDs, both of which contain very useful information on the 3D structure of the nucleon as well as the spin and OAM of the quarks in the nucleon. In [1] the authors introduced 5-D reduced Wigner distributions in the infinite momentum frame, or in light-front framework, which are functions of three momentum and two position variables. Working in the lightfront formalism is useful as the transverse boosts are Galilean or do not involve dynamics and longitudinal boosts are scale transformations. Thus it is possible to have a boost invariant description of the Wigner distributions in terms of lightfront wave functions. In [1] the Wigner distributions were calculated in light-cone constituent quark model and light-cone chiral quark-soliton model. Both these models have no gluonic degrees of freedom. In this work [2], we calculate the Wigner functions for a dressed quark in lightfront Hamiltonian perturbation theory, which is basically a relativistic composite spin $1 / 2$ state. This is a simple model with a gluonic degree of freedom. The state in expanded in Fock space in terms of multiparton light-front wave functions (LFWFs). The advantage is that this approach gives a field theoretic description of deep inelastic scattering processes and at the same time keeps 
close contact with parton model, the partons now are field theoretic, they are massive, non-collinear and interacting. To obtain the non-perturbative LFWFs for a bound state like the nucleon one needs a model light-front Hamiltonian. However, for a quark state dressed with a gluon the twobody light-front wave function can be obtained analytically. In the next section, we present a calculation of the Wigner distributions in this model.

\section{Wigner Distributions}

The Wigner distribution of quarks can be defined as $[1,3]$

$$
\begin{array}{r}
\rho^{[\Gamma]}\left(b_{\perp}, k_{\perp}, x, \sigma\right)=\int \frac{d^{2} \Delta_{\perp}}{(2 \pi)^{2}} e^{-i \Delta_{\perp} . b_{\perp}} \\
W^{[\Gamma]}\left(\Delta_{\perp}, k_{\perp}, x, \sigma\right) ;
\end{array}
$$

where $\Delta_{\perp}$ is momentum transfer from the initial state to the final state in transverse direction and $b_{\perp}$ is 2 dimensional vector in impact parameter space conjugate to $\Delta_{\perp}$. $W^{[\Gamma]}$ is the quark-quark correlator given by

$$
\begin{aligned}
& W^{[\Gamma]}\left(\Delta_{\perp}, k_{\perp}, x, \sigma\right)= \frac{1}{2} \int \frac{d z^{-} d^{2} z_{\perp}}{(2 \pi)^{3}} \\
& e^{i\left(x p^{+} z^{-} / 2-k_{\perp} z_{\perp}\right)} \\
&\left.\left\langle p^{+}, \frac{\Delta_{\perp}}{2}, \sigma\left|\bar{\psi}\left(-\frac{z}{2}\right) \Omega \Gamma \psi\left(\frac{z}{2}\right)\right| p^{+},-\frac{\Delta_{\perp}}{2}, \sigma\right\rangle\right|_{z^{+}=0}
\end{aligned}
$$

We take the target state to be a quark dressed with a gluon. We use the symmetric frame [4] where $p^{+}$and $\sigma$ define the longitudinal momentum of the target state and its helicity respectively. $x=k^{+} / p^{+}$is the fraction of longitudinal momentum of the dressed quark carried by the quark. $\Omega$ is the gauge link needed for color gauge invariance. Here, we use the light-front gauge, $A^{+}=0$ and take the gauge link to be unity. In fact the quark orbital angular momentum in this model does not depend on the gauge link. The symbol $\Gamma$ represents the Dirac matrix structure. The state of momentum $p$ and helicity $\sigma$, can be expanded in Fock space using the multi-parton light-front wave functions (LFWFs) [5]. The boost invariant two-particle LFWFs be calculated perturbatively as [5]. We use the two component formalism [6]. The quark state dressed with a gluon as we consider here mimics the bound state of a spin-1/2 particle and a spin-1 particle. However, for such a bound state the bound state mass $M$ should be less than the sum of the masses of the constituents for stability. In this work, we use the same mass for the bare as well as the dressed quark in perturbation theory [5]. The Wigner distributions can be expressed as overlaps of two-particle LFWFs. We take the momentum transfer to be completely in the transverse direction. In this case, the overlaps are diagonal or between two-particle LFWFs.

Wigner distributions of quarks with longitudinal polarization $\lambda$ in a target with longitudinal polarization $\Lambda$ is denoted by $\rho_{\Lambda \lambda}\left(\vec{b}_{\perp}, \vec{k}_{\perp}, x\right)$. This can be decomposed in terms of four Wigner functions as defined below:

$$
\begin{aligned}
\rho_{U U}\left(\vec{b}_{\perp}, \vec{k}_{\perp}, x\right)= & \frac{1}{2}\left[\rho^{\left[\gamma^{+}\right]}\left(\vec{b}_{\perp}, \vec{k}_{\perp}, x,+\vec{e}_{z}\right)\right. \\
& \left.+\rho^{\left[\gamma^{+}\right]}\left(\vec{b}_{\perp}, \vec{k}_{\perp}, x,-\vec{e}_{z}\right)\right]
\end{aligned}
$$

is the Wigner distribution of unpolarized quarks in unpolarized target state.

$$
\begin{aligned}
\rho_{L U}\left(\vec{b}_{\perp}, \vec{k}_{\perp}, x\right)= & \frac{1}{2}\left[\rho^{\left[\gamma^{+}\right]}\left(\vec{b}_{\perp}, \vec{k}_{\perp}, x,+\vec{e}_{z}\right)\right. \\
& \left.-\rho^{\left[\gamma^{+}\right]}\left(\vec{b}_{\perp}, \vec{k}_{\perp}, x,-\vec{e}_{z}\right)\right]
\end{aligned}
$$

is the distortion due to longitudinal polarization of the target state.

$$
\begin{aligned}
\rho_{U L}\left(\vec{b}_{\perp}, \vec{k}_{\perp}, x\right)= & \frac{1}{2}\left[\rho^{\left[\gamma^{+} \gamma_{5}\right]}\left(\vec{b}_{\perp}, \vec{k}_{\perp}, x,+\vec{e}_{z}\right)\right. \\
& \left.+\rho^{\left[\gamma^{+} \gamma_{5}\right]}\left(\vec{b}_{\perp}, \vec{k}_{\perp}, x,-\vec{e}_{z}\right)\right]
\end{aligned}
$$

is the distortion due to the longitudinal polarization of quarks, and

$$
\begin{aligned}
\rho_{L L}\left(\vec{b}_{\perp}, \vec{k}_{\perp}, x\right)= & \frac{1}{2}\left[\rho^{\left[\gamma^{+} \gamma_{5}\right]}\left(\vec{b}_{\perp}, \vec{k}_{\perp}, x,+\vec{e}_{z}\right)\right. \\
& \left.-\rho^{\left[\gamma^{+} \gamma_{5}\right]}\left(\vec{b}_{\perp}, \vec{k}_{\perp}, x,-\vec{e}_{z}\right)\right]
\end{aligned}
$$

is the distortion due to the correlation between the longitudinal polarized target state and quarks. $+\overrightarrow{e_{z}}$ and $-\overrightarrow{e_{z}}$ denote the helicity up and down of the target state, respectively. In our model, $\rho_{L U}=\rho_{U L}$. 
Using the two-particle LFWF the above distributions can be calculated analytically for a quark state dressed with a gluon [2]. In Figs. 1 and 2 we have shown the 3D plots for the Wigner distribution $\rho_{U U}$. In the numerical calculation for Eq.3 we have upper cut-off's $\Delta_{x}^{\max }$ and $\Delta_{y}^{\max }$ for the $\Delta_{\perp}$ integration. In all plots we have taken $m=0.33$ $\mathrm{GeV}$ and integrated over $x$. We have plotted $\rho_{U U}$ in $b$ space with $k_{\perp}=0.4 \mathrm{GeV}$ such that $\overrightarrow{k_{\perp}}=k \hat{j}$. The plot has a peak centered at $b_{x}=b_{y}=0$ decreasing in the outer regions of the $b$ space. The asymmetry in $b$ space can be understood from semi-classical arguments in a model with confinement. As no confining potential is present in our perturbative model, the behavior is expected to be different. In Figs. 3 and 4 we show the 3D plots for the Wigner distribution $\rho_{L U}$. This is the distortion of the Wigner distribution of unpolarized quarks due to the longitudinal polarization of the nucleon. We have shown $\rho_{L U}$ in $b$ space with $k_{\perp}=0.4 \mathrm{GeV}$ such that $\overrightarrow{k_{\perp}}=k \hat{j}$ for $\Delta_{\perp}^{\max }=1.0$ $\mathrm{GeV}$ and $\Delta_{\perp}^{\max }=5.0 \mathrm{GeV}$ respectively. Similar to [1] we observe a dipole structure in these plots and the dipole magnitude increases with increase in $\Delta_{\max }$.

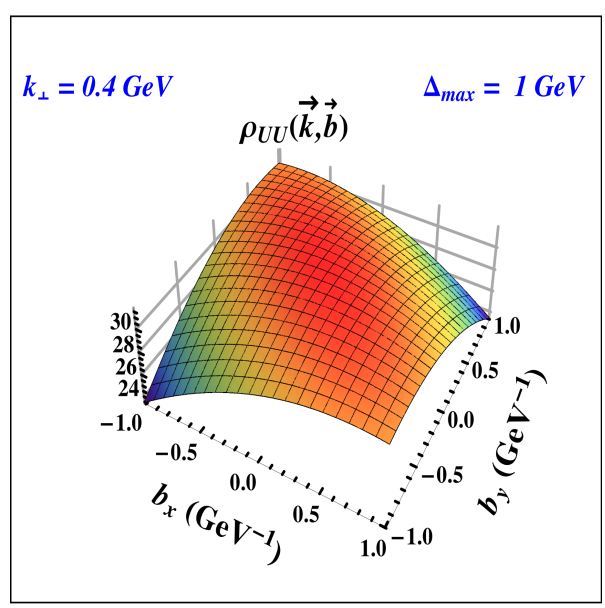

Figure 1. (Color online) 3D plots of the Wigner distributions $\rho_{U U}$ in $b$ space for $\Delta_{\perp}^{\max }=1.0 \mathrm{GeV}$ with $k_{\perp}=0.4 \mathrm{GeV}$. For all the plots we kept $m=0.33 \mathrm{GeV}$, integrated out the $x$ variable and we took $\overrightarrow{k_{\perp}}=k \hat{j}$ and $\overrightarrow{b_{\perp}}=b \hat{j}$.

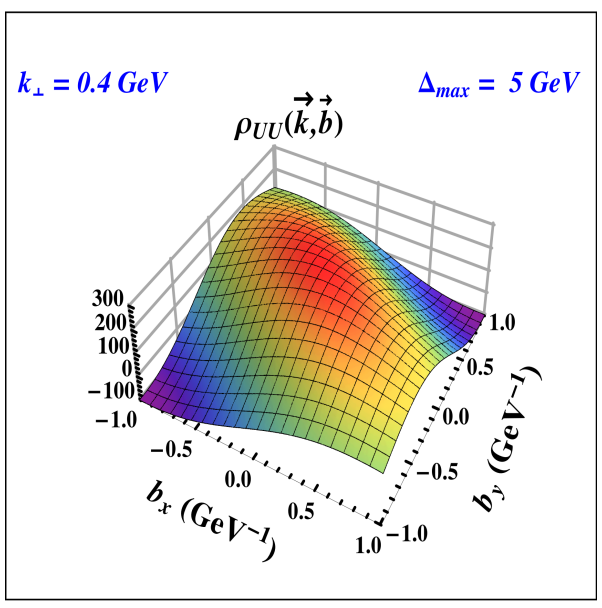

Figure 2. (Color online) 3D plots of the Wigner distributions $\rho_{U U}$ in $b$ space for $\Delta_{\perp}^{\max }=5.0 \mathrm{GeV}$ with $k_{\perp}=0.4 \mathrm{GeV}$.

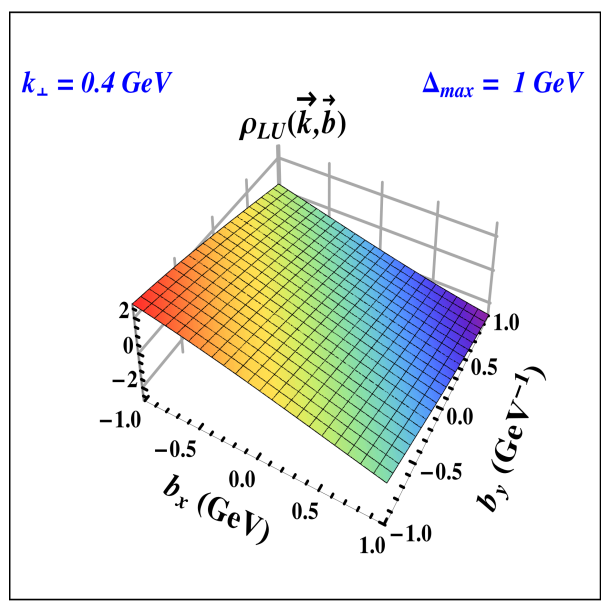

Figure 3. (Color online) 3D plots of the Wigner distributions $\rho_{L U}$ in $b$ space for $\Delta_{\perp}^{\max }=1.0 \mathrm{GeV}$ with $k_{\perp}=0.4 \mathrm{GeV}$.

\section{Orbital Angular Momentum of the quarks}

The quark-quark correlator in Eq.(2) defining the Wigner distributions can be parameterized in terms of generalized transverse momentum dependent parton distributions (GTMDs) [3]. For the twist two case we have eight GTMDs as defined below : 


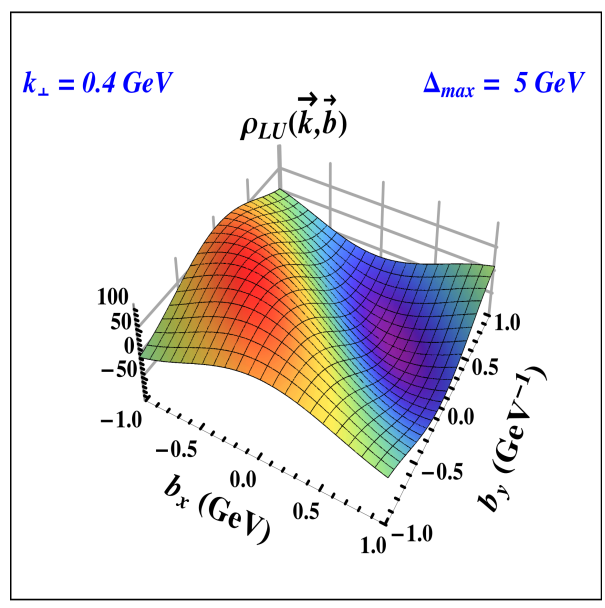

Figure 4. (Color online) 3D plots of the Wigner distributions $\rho_{L U}$ in $b$ space for $\Delta_{\perp}^{\max }=5.0 \mathrm{GeV}$ with $k_{\perp}=0.4 \mathrm{GeV}$.

$$
\begin{gathered}
W_{\lambda, \lambda^{\prime}}^{\left[\gamma^{+}\right]}=\frac{1}{2 M} \bar{u}\left(p^{\prime}, \lambda^{\prime}\right)\left[F_{1,1}-\frac{i \sigma^{i+} k_{i \perp}}{P^{+}} F_{1,2}\right. \\
\left.-\frac{i \sigma^{i+} \Delta_{i \perp}}{P^{+}} F_{1,3}+\frac{i \sigma^{i j} k_{i \perp} \Delta_{j \perp}}{M^{2}} F_{1,4}\right] u(p, \lambda) ; \\
W_{\lambda, \lambda^{\prime}}^{\left[\gamma^{+} \gamma_{5}\right]}=\frac{\bar{u}\left(p^{\prime}, \lambda^{\prime}\right)}{2 M}\left[\frac{-i \epsilon_{\perp}^{i j} k_{i \perp} \Delta_{j \perp}}{M^{2}} G_{1,1}\right. \\
-\frac{i \sigma^{i+} \gamma_{5} k_{i \perp}}{P^{+}} G_{1,2}-\frac{i \sigma^{i+} \gamma_{5} \Delta_{i \perp}}{P^{+}} G_{1,3} \\
\left.+i \sigma^{+-} \gamma_{5} G_{1,4}\right] u(p, \lambda) .
\end{gathered}
$$

The GTMDs can be calculated analytically in the dressed quark model. Using the relation between the GTMD $F_{14}$ and the canonical OAM $[1,7,8]$ :

$$
l_{z}^{q}=-\int d x d^{2} k_{\perp} \frac{k_{\perp}^{2}}{m^{2}} F_{14}
$$

The canonical OAM in this model is given by [2]:

$$
\begin{array}{r}
l_{z}^{q}=-2 N \int d x\left(1-x^{2}\right)\left[I_{1}-\right. \\
\left.m^{2}(x-1)^{2} I_{2}\right]
\end{array}
$$

On the other hand, the kinetic quark OAM is given in terms of the GPDs as :

$$
\begin{array}{r}
L_{z}^{q}=\frac{1}{2} \int d x\left\{x\left[H^{q}(x, 0,0)+E^{q}(x, 0,0)\right]\right. \\
\left.-\tilde{H}^{q}(x, 0,0)\right\} .
\end{array}
$$

In the model considered here, this becomes,

$$
\begin{array}{r}
L_{z}^{q}=\frac{N}{2} \int d x\left\{-f(x) I_{1}\right. \\
\left.+4 m^{2}(1-x)^{2} I_{2}\right\}
\end{array}
$$

where,

$$
\begin{aligned}
I_{1} & =\int \frac{d^{2} k_{\perp}}{m^{2}(1-x)^{2}+\left(k_{\perp}\right)^{2}} \\
& =\pi \log \left[\frac{Q^{2}+m^{2}(1-x)^{2}}{\mu^{2}+m^{2}(1-x)^{2}}\right] ; \\
I_{2} & =\int \frac{d^{2} k_{\perp}}{\left(m^{2}(1-x)^{2}+\left(k_{\perp}\right)^{2}\right)^{2}} \\
& =\frac{\pi}{\left(m^{2}(1-x)^{2}\right)} ; \\
f(x) & =2\left(1+x^{2}\right) .
\end{aligned}
$$

$Q$ is the large scale involved in the process, which comes from the large momentum cutoff in this approach [5]. $\mu$ can be safely taken to be zero provided the quark mass is non-zero. Similar qualitative behavior of $L_{z}^{q}$ and $l_{z}^{q}$ are observed, both giving negative values. However the magnitude of the two OAM differs in our model, unlike the case in [1], where these were calculated in several models without any gluonic degrees of freedom and the total quark contribution to the OAM were equal for both cases. Thus one can see the effect of the gluonic degrees of freedom to the OAM in the model considered here. The contribution from the gluons has been calculated recently also in [9] in this model.

\section{Conclusion}

We presented a recent calculation of the Wigner distributions for a quark state dressed with a gluon using the overlap representation in terms of the LFWFs. This is a relativistic composite spin$1 / 2$ system which has a gluonic degree of freedom. The Wigner distributions are calculated both for unpolarized and longitudinally polarized 
target and quarks and the correlations are shown in transverse position space. The kinetic quark OAM using the GPD sum rule and the canonical OAM were also calculated in this model and it was shown that these are different in magnitude, the difference is an effect of the gluonic degree of freedom.

\section{Acknowledgements}

This work is supported by the DST project SR/S2/HEP029/2010, Govt. of India. AM thanks the organizers of Transversity 2014, June 9-13, 2014, Chia, Sardinia for the invitation.

\section{References}

[1] C. Lorce, B.Pasquini, Phys. Rev. D84, 014015 (2011).
[2] A. Mukherjee, S. Nair, V. K. Ojha, Phys. Rev. D D90, 014024 (2014).

[3] S.Meissner, A.Metz, and M. Schlegel, JHEP 08 (2009) 056; S.Meissner, A.Metz, M. Schlegel and K. Goeke, JHEP 08 (2008) 038.

[4] S.J.Brodsky, M.Diehl, D.S.Hwang, Nucl. Phys. B596, (2001) 99.

[5] A. Harindranath and R. Kundu, Phys. Rev. D59, 116013.

[6] W-M. Zhang and A. Harindranath, Phys. Rev. D48, 4881 (1993).

[7] Y. Hatta, Phys. Lett. B 708, 186 (2012).

[8] C. Lorce, B. Pasquini, X. Xiong, F. Yuan, Phys. Rev. D 85, 114006 (2012).

[9] K. Kanazawa, C. Lorce, A. Metz, B. Pasquini, M. Schlegel, Phys. Rev. D 90, 014028 (2014). 